\title{
Genetische Untersuchungen beim Menschen ab dem 1. April 2007 gesetzlich geregelt
}

\author{
Der Bundesrat setzt auf den 1. April 2007 das Gesetz über genetische Unter- \\ suchungen beim Menschen (GUMG) zusammen mit zwei Verordnungen in \\ Kraft und ernennt eine Expertenkommission. Auf das gleiche Datum tritt \\ zudem eine einschlägige Verordnung des EDI in Kraft. Gesetz und Verordnun- \\ gen regeln die wesentlichen Aspekte der Durchführung genetischer Unter- \\ suchungen beim Menschen mit dem Ziel, die Menschenwürde zu schützen, \\ Missbräuche zu verhindern und die Qualität der Untersuchungen sicher- \\ zustellen.
}

Cristina Benedetti

\footnotetext{
Untersuchungen zu Forschungszwecken unterliegen aber den Regelungen im Bereich der Forschung.
}

Korrespondenz:

Cristina Benedetti,

eidg. Dipl. pharm. MPH

BAG

Direktionsbereich Öffentliche

Gesundheit

Abteilung Biomedizin

CH-3003 Bern

cristina.benedetti@bag.admin.ch
Genetische Untersuchungen beim Menschen erweitern das Spektrum von diagnostischen, präventiven und therapeutischen Möglichkeiten bisher unheilbarer Krankheiten. Sie ermöglichen es zudem, Krankheitsveranlagungen vor dem Auftreten klinischer Symptome zu entdecken oder genetische Defekte bereits vorgeburtlich festzustellen. Sie werfen deshalb heikle ethische, psychologische und soziale Fragen auf. Das Wissen um genetische Veranlagungen kann nicht nur präventiv genutzt werden, sondern kann auch schwierige Fragen bezüglich der Lebensund Familienplanung aufwerfen. Ebenso könnte dieses Wissen durch Dritte missbraucht werden, z.B. durch Ausschluss der betroffenen Person von Versicherungsleistungen oder Arbeitsverhältnissen. Das Bundesgesetz über genetische Untersuchungen beim Menschen, das am 1. April 2007 in Kraft tritt, regelt die folgenden Bereiche:

- die Anwendung genetischer Untersuchungen beim Menschen zu medizinischen Zwecken und im Arbeits-, Versicherungs- und Haftpflichtbereich;

- die Erstellung von DNA-Profilen zur Klärung der Abstammung oder zur Identifizierung von Personen, mit Ausnahme der Verwendung von DNA-Profilen im Strafverfahren und zur Identifizierung von unbekannten oder vermissten Personen (auf diese ist das DNAProfil-Gesetz anwendbar).

Das Gesetz bezweckt, die Menschenwürde zu schützen, Missbräuche zu verhindern und die
Qualität der Untersuchungen sicherzustellen. In den Anwendungsbereich des Gesetzes fallen zyto- und molekulargenetische Untersuchungen zur Abklärung ererbter oder während der Embryonalphase erworbener Eigenschaften des Erbguts des Menschen sowie alle weiteren Laboruntersuchungen, die unmittelbar darauf abzielen, solche Informationen über das Erbgut zu erhalten. Im Zusammenhang mit pränatalen Untersuchungen werden unter den pränatalen Risikoabklärungen auch Untersuchungen des Embryos mit bildgebenden Verfahren erfasst. Nicht den Bestimmungen des Gesetzes unterliegen hingegen Untersuchungen von im Laufe des Lebens erworbenen Eigenschaften (somatische Mutationen) sowie genetische Untersuchungen zu Forschungszwecken. Diese Untersuchungen sind daher nicht bewilligungspflichtig*.

\section{Verordnete Informationspflicht}

Im Zusammenhang mit genetischen Untersuchungen hält das Gesetz fest, dass niemand wegen seines Erbgutes diskriminiert werden darf (Diskriminierungsverbot). Zudem müssen betroffene Personen ihre Zustimmung zur Untersuchung geben und haben das Recht auf Nichtwissen. Das Gesetz legt ausserdem grosses Gewicht auf die Qualitätssicherung, denn genetische Untersuchungen sind komplex, ihre Ergebnisse schwierig zu interpretieren, und die Konsequenzen können für die Betroffenen und ihre Angehörigen schwerwiegend sein. Hohe Qualität wird daher nicht nur bei der Durchführung der Untersuchungen durch die Laboratorien gefor- 
Die Mitglieder der Expertenkommission Sabina Gallati, Präsidentin (Bern); Armand Bottani (Genf); Walter Bär (Zürich); Matthias Baumgartner (Zürich); Gieri Cathomas (Liestal); Bernice Elger (Genf); Susan Gerber (Bern); Andreas Huber (Aarau); Peter Miny (Basel); Michael Morris (Genf); Judith Pók Lundquist (Zürich); Nicole Probst-Hensch (Zürich).

dert, sondern auch bei der Information und der Beratung der betroffenen Personen. So legt das Gesetz fest, über welche Aspekte die betroffene Person bei einer präsymptomatischen oder pränatalen genetischen Untersuchung im Rahmen einer nichtdirektiven, fachkundigen Beratung informiert werden muss. Das Gesetz verpflichtet zudem die Kantone, unabhängige Informationsund Beratungsstellen für pränatale Untersuchungen zur Verfügung zu stellen.

\section{Qualität dank Qualifikation}

Nur vom Bundesamt für Gesundheit (BAG) bewilligte Laboratorien dürfen genetische Untersuchungen durchführen. Kernelemente der Bewilligung sind die Qualifikation der Laborleiterin oder des Laborleiters, ein Qualitätsmanagementsystem und eine regelmässige externe Qualitätskontrolle. Die berufliche Qualifikation bzw. der Titel der Leiterin oder des Leiters eines Laboratoriums hat Auswirkungen auf das Spektrum der genetischen Untersuchungen, welche das Laboratorium durchführen darf. Ein Laboratorium mit einer Leiterin oder einem Leiter, die oder der den umfassenden Titel «Spezialistin/Spezialist für medizinisch-genetische Analytik FAMH» besitzt, ist zur Durchführung sämtlicher genetischer Untersuchungen befugt. Ein Laboratorium darf hingegen nur einzelne, auf Verordnungsstufe definierte genetische Untersuchungen durchführen, wenn die zur Laborleitung designierte Person über einen Titel als Spezialistin/ Spezialist für klinisch-chemische Analytik FAMH, hämatologische Analytik FAMH, klinisch-immunologische Analytik FAMH, labormedizinische Analytik FAMH (pluridisziplinär) oder Fachärztin/Facharzt für Pathologie FMH mit Schwerpunkt Molekularpathologie verfügt. Die Liste der Untersuchungen wird durch das Eidg. Departement des Innern in Anlehnung an die im Bereich der obligatorischen Krankenversicherung geltende Regelung festgelegt. Mit angemeldeten und gegebenenfalls unangemeldeten Inspektionen wird die Einhaltung der Bestimmungen überprüft. Für akkreditierte
Laboratorien ersetzen die Nachkontrollen der schweizerischen Akkreditierungsstelle die Inspektionen. So werden kostspielige Doppelspurigkeiten vermieden.

Bewilligungsvoraussetzungen und -verfahren sowie die Pflichten der Laboratorien sind in der Verordnung über genetische Untersuchungen beim Menschen festgelegt. Die Laboratorien müssen bis zum 30. Juni 2007 ihr Gesuch beim BAG einreichen.

\section{Reihenuntersuchungen}

Reihenuntersuchungen im Sinne des Gesetzes sind genetische Untersuchungen, die systematisch der gesamten Bevölkerung oder bestimmten Bevölkerungsgruppen angeboten werden. Damit sie durchgeführt werden können, muss das Anwendungskonzept durch das BAG bewilligt sein. Das Gesetz definiert drei Voraussetzungen für die Erteilung einer solchen Bewilligung: - die Möglichkeit einer Frühbehandlung oder einer Prophylaxe;

- zuverlässige Ergebnisse der Untersuchungsmethode;

- Sicherstellung einer angemessenen genetischen Beratung.

Bei den Gesuchstellenden handelt es sich beispielsweise um medizinische Fachgesellschaften, kantonale Gesundheitsbehörden oder Universitätsspitäler. Reihenuntersuchungen, die beim Inkrafttreten des Gesetzes bereits durchgeführt werden, sind von der Bewilligungspflicht ausgenommen.

\section{Erstellung von DNA-Profilen}

Das Gesetz schreibt eine obligatorische Anerkennung der Laboratorien vor, die DNA-Profile zur Klärung der Abstammung oder zur Identifizierung ausserhalb von Strafverfahren erstellen. Die Voraussetzungen und das Verfahren zur Anerkennung sind in der Verordnung über die Erstellung von DNA-Profilen im Zivil- und im Verwaltungsbereich geregelt. Zuständig für die Anerkennung ist das Eidgenössische Justiz- und Polizeidepartement. Auskünfte erteilt das Bundesamt für Polizei.

\section{Expertenkommission}

Gleichzeitig mit der Inkraftsetzung von Gesetz und Verordnungen setzt der Bundesrat auf den 1. April 2007 die Expertenkommission für genetische Untersuchungen beim Menschen ein. Die Kommission hat zur Aufgabe, wissenschaftliche und praktische Entwicklungen im Bereich der genetischen Untersuchungen aufmerksam zu verfolgen, Empfehlungen dazu abzugeben und 
rechtzeitig auf Probleme und Lücken in der Gesetzgebung aufmerksam zu machen. Sie besteht aus 12 Mitgliedern mit fundierten Kenntnissen und Erfahrung in Bereichen wie Humangenetik, Kinder- und Frauenheilkunde, Rechtsmedizin, Analytik und Molekularpathologie.

\section{Weitere Informationen}

Für die Bewilligung zur Durchführung von genetischen Untersuchungen und Reihenuntersuchungen: Bundesamt für Gesundheit, Direktionsbereich Öffentliche Gesundheit, Abteilung Biomedizin, 3003 Bern, Tel. 0313235154 (Sekretariat), Fax 0313226233.

E-Mail: genetictesting@bag.admin.ch, Internet: www.bag.admin.ch/genetictesting.
Für die Anerkennung von Laboratorien zur Erstellung von DNA-Profilen: Bundesamt für Polizei, Abteilung Dienste, Anerkennungsstelle VDZV, 3003 Bern, Fax 03132414 10, Internet: www.fedpol.admin.ch/fedpol/de/home/dienst leistungen.html.

\section{Informationsseite}

Das Bundesamt für Gesundheit und das Bundesamt für Polizei werden ab dem 1. April 2007 eine Seite für den Vollzug aufschalten. Gesuchstellerinnen und Gesuchsteller werden hier die für sie wichtigsten Informationen sowie Formulare für das Einreichen von Gesuchen abrufen können. 